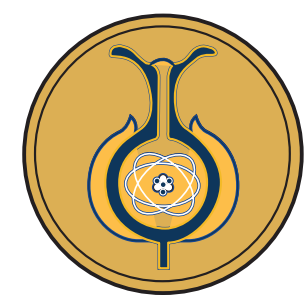

\title{
Descifrando el clima espacial: ciclos solares y ondas magnéticas de Rossby
}

\author{
Understanding Space Weather: Solar Cycles and Magnetic Rossby Waves
}

Decifrando o clima espacial: ciclos solares e ondas magnéticas de Rossby

\author{
Xiomara Márquez-Artavia \\ xiomara.marquez.artavia@una.cr \\ Departamento de Física \\ Universidad Nacional \\ Heredia, Costa Rica \\ https://orcid.org/0000-0002-4905-6123
}

Recibido-Received: 23/ago/2018 / Corregido-Corrected: 20/ene /2019

Aceptado-Accepted: 4/feb/2019/Publicado-Published: 31/jul/2019

\section{Resumen}

El objetivo de esta investigación es explorar si existe una relación entre las ondas magnéticas de Rossby rápidas o lentas y ciertas observaciones de la actividad solar de largo y muy largo plazo (meses, años, décadas...). El artículo intenta mostrar si los periodos de esas ondas y sus características corresponden a los valores de duración los ciclos de Rieger, Haly, 0 bien cuasiperiodos de llamaradas y eyecciones de masa coronal. Se inicia con una revisión bibliográfica y un resumen de los resultados de una tesis doctoral que resuelve completamente el modelo matemático en cuestión, con las ecuaciones derivadas de ese trabajo previo, se calcula los periodos de las ondas que serán comparados con las observaciones. Estos resultados teóricos sugieren que cierta actividad solar podría deberse a las ondas magnéticas de Rossby, originadas en el interior del Sol, específicamente en la tacoclina. Las ondas lentas se proponen ligadas a las periodicidades de largo y muy largo plazo y las ondas rápidas de Rossby se asocian con periodicidades de unos años a meses. El estudio de estas perturbaciones de Rossby y sus propiedades, sin duda, va a ayudar a comprender el origen y las características del campo magnético del Sol.

Palabras claves: Ciclo solar; ondas magnéticas de Rossby; tacoclina; ondas magnetohidrodinámicas; física solar; clima espacial

\section{Abstract}

The objective of this investigation is to explore the relationship between slow and fast magnetic Rossby waves and certain observations of long-term solar activity (months, years, decades, ...). This paper seeks to determine if the periods of these waves correspond to the values recorded for cycles such as the Rieger cycle 
and the Haly cycle, or otherwise to quasi-periodic outbursts and of flares and Coronal Mass Ejections. The study begins with a literature review and a summary of the results of a Ph.D. dissertation that completely solves the mathematical model analyzed; using the equations derived from this previous work, the periods of the waves are calculated and then compared to observations. The results of this comparison suggest that certain types of solar activity may be due to Rossby magnetic waves originating within the Sun, specifically in the tachocline. The slow magnetic Rossby waves are associated with long-term and very long-term cycles, while the fast magnetic Rossby waves are a plausible cause for cycles with periods of months or a few years. The study of magnetic Rossby waves and their properties will doubtlessly provide new insights into the origin and properties of the solar magnetic field.

Keywords: Solar cycle; slow and fast magnetic Rossby waves; tachocline; MHD waves; solar physics, space weather.

\section{Resumo}

0 objetivo desta pesquisa é explorar se existe uma relação entre as ondas magnéticas de Rossby rápidas ou lentas e certas observações de atividade solar de longo e muito longo prazo (meses, anos, décadas...). 0 artigo tenta mostrar se os períodos dessas ondas e suas características correspondem aos valores de duração dos ciclos de Rieger, Hale, ou quase períodos de erupções solares e ejeções de massa coronal. Ele começa com uma revisão bibliográfica e um resumo dos resultados de uma tese de doutorado que resolve completamente o modelo matemático em questão, com as equações derivadas desse trabalho prévio, calcula-se os períodos das ondas que serão comparados com as observações. Estes resultados teóricos sugerem que certa atividade solar poderia derivar das ondas magnéticas de Rossby, originadas no interior do Sol, especificamente na tacoclina. Ondas lentas são propostas ligadas às periodicidades de longo e muito longo prazo e as ondas rápidas de Rossby estão associadas a periodicidades de alguns anos a meses. 0 estudo desses distúrbios de Rossby e suas propriedades, sem dúvida, ajudará a entender a origem e as características do campo magnético do Sol.

Palavras-chave: Ciclo solar; ondas magnéticas de Rossby; tacoclina; ondas magneto-hidrodinâmicas; física solar; clima espacial.

$\mathrm{D}$ el Sol nos llega prácticamente toda la energía que disponemos, a esta se debe la vida sobre la Tierra. La energía en forma de luz y calor que proviene del Sol produce cambios en la atmósfera, además de generar, directamente, la evaporación: una etapa fundamental en el ciclo hidrológico. El efecto invernadero y su consecuente calentamiento global es también dependiente del calor generado por el astro. En el sentido biológico las plantas, algas y otros organismos aprovechan la luz solar para realizar la fotosíntesis, al formar agua, oxígeno y glucosa, contenida en los frutos. Por lo tanto, la actividad solar, principalmente a largo plazo, está ligada con la cantidad de lluvias, las cosechas, y la temperatura del planeta: estos afectan de forma directa la vida en el planeta y las actividades económicas (Lang, 1995).

Para el ser humano como ente biológico, la luz solar ayuda a producir la vitamina D, necesaria para el correcto funcionamiento del cuerpo y la formación de los huesos. La ausencia de sol en los países de latitudes más altas produce una enfermedad 
denominada SAD, la depresión estacional. Por estas razones al estudiar su comportamiento, se intenta predecir cualquier fenómeno, que pueda afectar la Tierra.

Nuestra estrella es considerada un astro promedio, tipo $\mathrm{G}$ de la secuencia principal, una enana amarilla en la mitad de su vida media. Este corresponde al $98 \%$ de la masa del Sistema Solar, aunque la estrella en sí sea más pequeña que el común. Está constituido de plasma, un gas con carga eléctrica, formado en su mayoría por hidrógeno $92.1 \%$ y helio $7.8 \%$ con trazas de otros elementos (Zeilik et al., 1987).

En su interior se encuentra el núcleo donde se produce la fusión nuclear que genera la energía de la estrella. Luego se localiza la zona radiactiva donde los fotones transportan la energía hacia las capas más externas, después está la zona de convección, donde las columnas de gas caliente suben y se enfrían y descienden de nuevo. La superficie solar que cubre su interior es una delgada capa, la más profunda de la atmósfera solar, denominada la fotosfera, y que visualizamos desde la Tierra. En la fotosfera, con su temperatura de aproximadamente 5500 $\mathrm{K}$, frecuentemente se observan las manchas solares, que por lo general, son indicadores del desarrollo de las actividades energéticas en la cromosfera y la corona solar.

La atmósfera solar está compuesta por la fotosfera, la cromosfera de unos $5000 \mathrm{~km}$ de espesor y la capa más externa: la corona. Esta es la que se observa a simple vista en un eclipse total del Sol, cuando la Luna cubre totalmente al astro. Su luz es tenue, pero se extiende muchos kilómetros en el espacio y tiene unas temperaturas extremadamente altas y fuertes campos magnéticos.

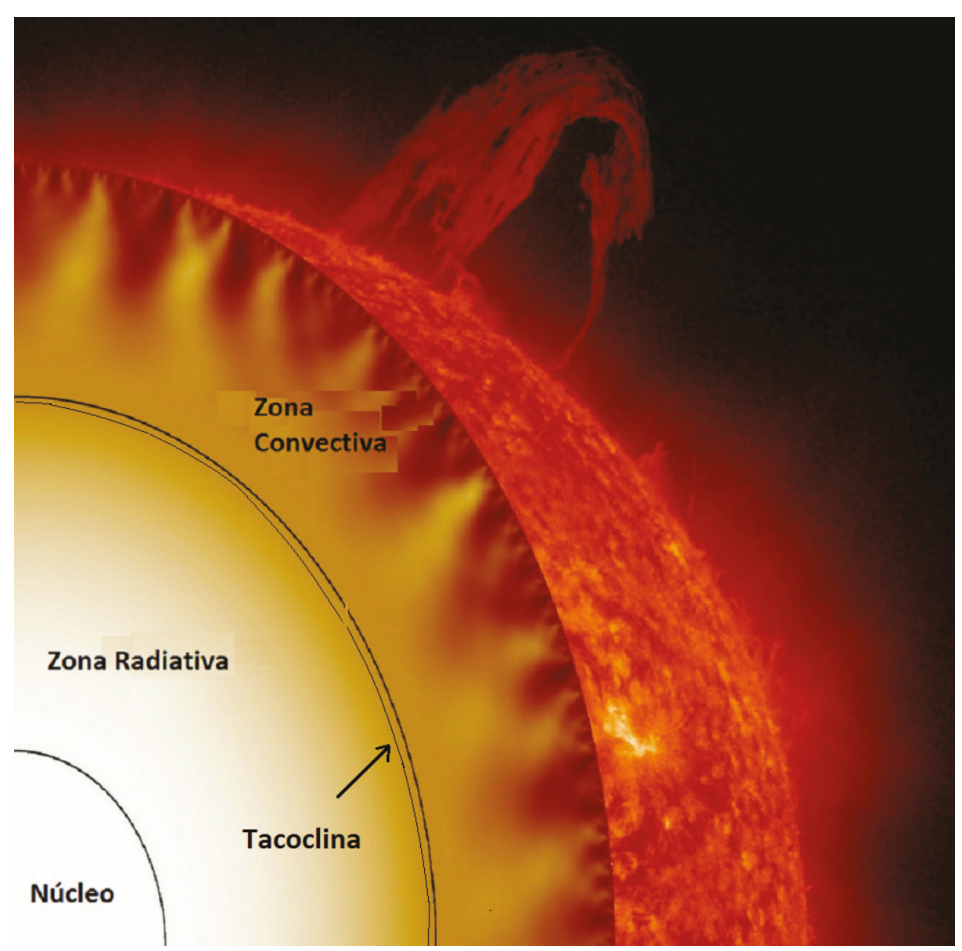

Figura 1. Interior del Sol, la tacoclina se encuentra entre la zona radiativa y la región de convección.

Extraído de: Imagen cortesía de la NASA/Marshall Solar Physics. 
El campo magnético solar ha sido objeto de mucha investigación, se considera que a gran escala, durante el mínimo de actividad, la componente axisimétrica del campo poloidal es, principalmente dipolar, con una magnitud de 10G para la fotosfera solar; sin embargo, cuando el ciclo avanza el campo muestra componentes multipolares y llega a ser más complicado (Miesch, 2005). Además, su estructura y propiedades sufren transiciones importantes con la altura (Solanski, et al., 2006).

Se considera que el mecanismo de dínamo que opera en la parte baja de la zona de convección es el origen del campo magnético solar, aunque actualmente muchos modelos colocan el dínamo en una zona de transición entre la región de convección y la zona radiativa, denominada tacoclina, ver la Figura 1 Esta delgada capa de transición se encuentra a una distancia de $\sim 0.7 \mathrm{R}_{\odot}$, y tiene un espesor medio de $\sim 0.04 \mathrm{R}_{\odot}$ (Hughes et al., 2007).

La región de convección rota diferencialmente, es decir, unas latitudes rotan a mayor velocidad que otras. El Sol rota más rápido en el ecuador, donde tarda 26 días en completar un giro, pero en los polos dura más de 30 días. La zona de radiación que es más interna gira como cuerpo rígido. $\mathrm{Al}$ ubicarse la tacoclina entre las dos regiones, una con rotación diferencial y otra de cuerpo rígido, existe un gran efecto de cizalla. De esta región se ocupa el presente artículo.

Muchos investigadores sugieren que la componente toroidal del campo magnético de la tacoclina es de mayor amplitud que la componente poloidal (Tobías, 2005). Otros autores apuntan a que debe de ser completamente toroidal (Gilman, 2000, Zaqarashvili et al., 2007).

El modelo matemático denominado "MHD ShallowWater" o la aproximación magnetohidrodinámica de la capa de fluido,
(Márquez-Artavia et al., 2017, Zaqarashvili et al., 2015, 2009), ha predicho la presencia de ondas en capas delgadas de fluido, como la tacoclina, y recientemente se ha sugerido que si estas ondas se generan en la tacoclina su efecto puede manifestarse en la superficie solar, (Dikpati et al., 2017).

Entonces ¿qué es una onda? Una onda es una perturbación que se propaga en forma periódica, es decir repetitiva. Su característica más importante es la frecuencia, es decir, el número de ciclos por segundo, conocer la frecuencia de una onda permite su clasificación y determinar la velocidad de propagación y su dirección.

Existen diferentes tipos de ondas en fluidos, entre ellas podemos mencionar: las de gravedad, las de Kelvin y las de Rossby. Las ondas de Rossby son comunes en los océanos y en la atmósfera (Kessler, 2006), son el efecto de la rotación del sistema, en ese caso la Tierra. La rotación produce una fuerza denominada Coriolis, y varía con la latitud, esta variación produce un movimiento neto del flujo hacia el oeste.

Las ondas de Rossby son el resultado de la ley de conservación del momento angular, o la vorticidad absoluta y su velocidad es proporcional a la velocidad de rotación del sistema. La presencia de ondas de Rossby en el Sol podría ser una acertada posibilidad, sin embargo, el campo magnético solar tiene una fuerte influencia sobre el movimiento del plasma, por contener carga eléctrica.

El efecto del campo magnético solar sobre estas ondas hace que aparezcan dos modos uno que viaja al oeste, muy similar en sus características al caso hidrodinámico y otro modo más lento que lo realiza hacia el este, con periodos más grandes, a estas denominaremos ondas magnéticas rápidas de Rossby y ondas magnéticas lentas de Rossby, respectivamente. 


\section{Características de las ondas magnéticas de Rossby}

Las propiedades físicas de las ondas magnéticas de Rossby han sido predichas matemáticamente por varios autores (Márquez-Artavia et al., 2017, Gilman et al., 2002, Zaqarashvili et al., 2015). Márquez-Artavia et al. (2017) han descrito exhaustivamente el comportamiento de las ondas magnéticas de Rossby en la presencia de un campo magnético tanto débil como fuerte y en un sistema tanto con rotación rápida como lenta, esto es un modelo que contempla un cascarón esférico de fluido conductor inmerso en un campo magnético de tipo toroidal, ver la Figura 2.

$\longrightarrow$ Dirección del Campo Magnético

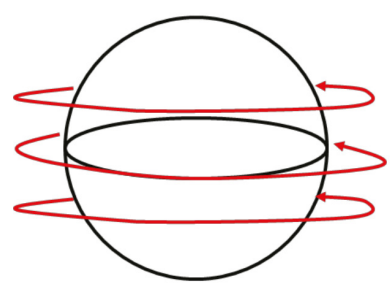

a) Toroidal

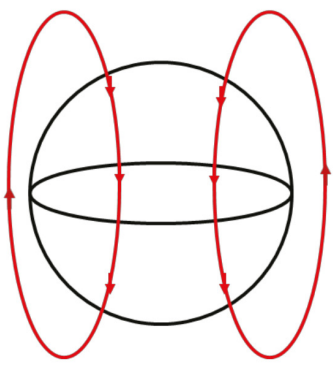

b) Poloidal
Figura 2. Dos configuraciones del campo magnético para una esfera de fluido, un campo magnético en general posee dos componentes, una toroidal y otra poloidal. Muchos investigadores consideran que la componente toroidal del campo magnético de la tacoclina es de mayor magnitud que la poloidal. Nota: Fuente propia del estudio.
Altura sin dimensiones de la capa fluida para una onda rápida magnética de Rossby
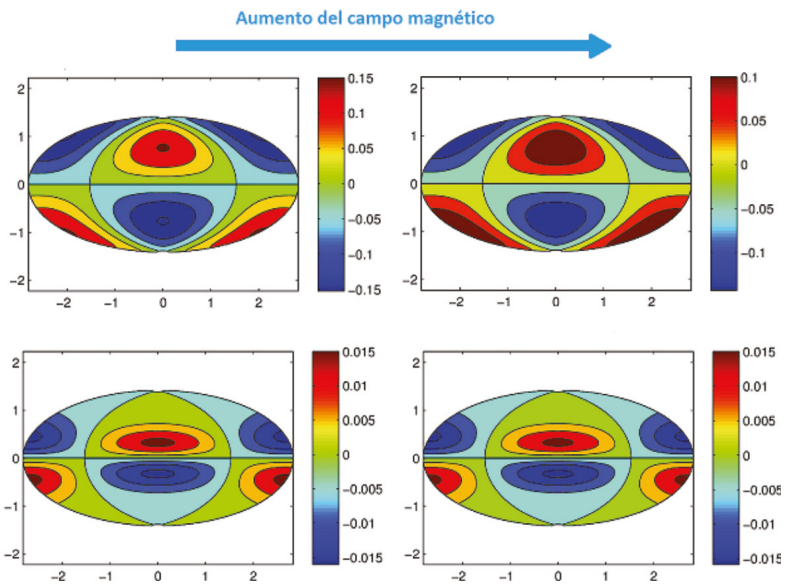

Figura 3. Confinamiento ecuatorial para una onda magnética rápida de Rossby, los cuadros muestran la altura sin dimensiones (dividida por un factor) de la capa de fluido al propagarse una onda de estas. Al incrementar la rotación y el campo magnético la onda restringe su movimiento a una banda de latitud cerca del ecuador.

Extraído de: Márquez-Artavia et al.(2017).

donde $\Omega_{0}$ es la velocidad de rotación del sistema, $n$ es un número entero $(1,2,3 \ldots)$ y m es también un número entero $(0,1,2, \ldots n)$. Se tiene entonces un conjunto de posibles ondas con diferentes velocidades y periodos. magnéticas rápidas de Rossby tienen periodos desde 26 días hasta unos años, con longitudes de onda grandes $(>1000 \mathrm{~km})$ y tienen la propiedad de que al aumentar el valor de la rotación del sistema o en la presencia de un campo magnético moderado se confinan ecuatorialmente, es decir su movimiento se restringe a la zona ecuatorial, ver la Figura 3. Su frecuencia según, Zaqarashvili et al. (2009), está dada por

$$
\omega=-\frac{2 \Omega_{o} m}{n(n+1)},(1)
$$


Las ondas magnéticas lentas de Rossby tienen periodos muy largos que van en rangos desde miles de años, siglos o decenios, dependiendo del valor del campo magnético. Esta onda es extremadamente sensible a la intensidad del campo magnético, dado a que a él debe su existencia. La relación entre el periodo y la intensidad del campo es inversamente proporcional, es decir, cuando el campo es más débil la onda presenta los periodos más largos y viceversa. Sin embargo, esta onda de Rossby no presenta confinamiento ecuatorial para ningún valor del campo magnético o la rotación, y esta propiedad es fundamental. La expresión para la frecuencia, según Zaqarashvilli et al. (2009), es

$\omega=\frac{B_{0}^{2}}{2 \rho \mu_{0} \Omega_{0} R_{0}^{2}}[n(n+1)-2]$,

siendo $B_{0}$ el campo magnético de la tacoclina, $\rho$ la densidad del fluido, $\mu_{0}$ la permeabilidad del espacio libre, que son en general valores constantes conocidos, exceptuando el valor del campo magnético que continúa siendo una interrogante.

Existe también una onda anómala lenta de Rossby que viaja hacia el oeste, con periodos de miles a millones de años, que depende de la intensidad del campo magnético. Esta onda solo existe por la influencia del campo magnético y como el resto de las ondas lentas de Rossby no experimenta confinamiento ecuatorial. Ha sido descrita matemáticamente por primera vez por Márquez-Artavia et al. (2017). La relación entre la frecuencia de la onda y el campo magnético, según Márquez-Artavia et al. (2017), es

$$
\omega=-\frac{B_{0}^{4}}{10 \rho^{2} \mu_{0}^{2} g H_{0} \Omega_{0} R_{0}^{2}}
$$

donde $g$ es el valor de la aceleración gravitacional y $H_{0}$ es la altura promedio de la capa. Como esta frecuencia es proporcional a la cuarta potencia del campo magnético, hace que su valor sea muy pequeño lo que provoca que la onda sea muy lenta, con periodos muy largos.

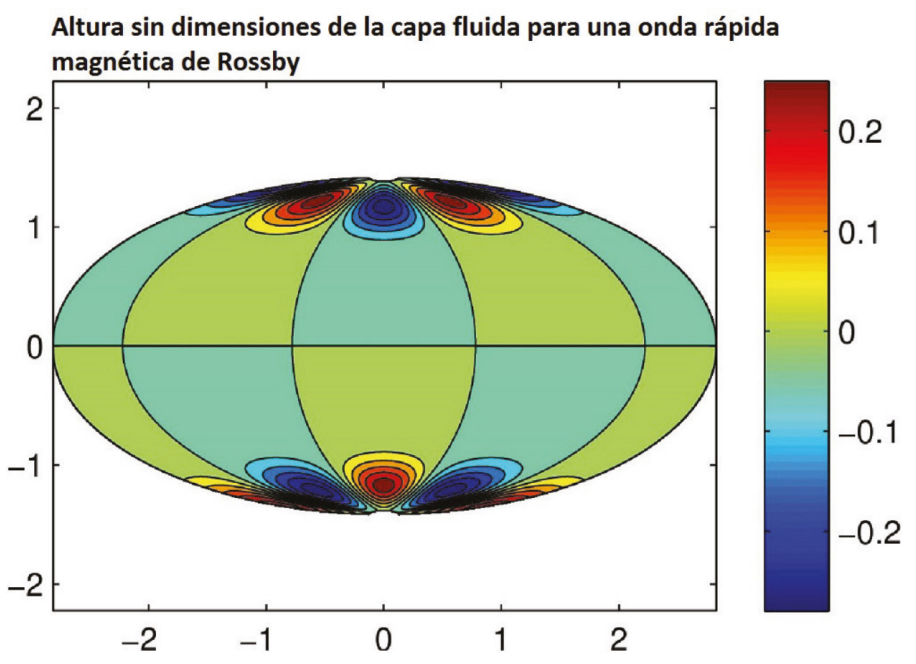

Figura 4. Confinamiento polar para una onda rápida magnética de Rossby cuando el campo magnético es fuerte, similar al que existe en los púlsares y magnetares.

Extraído de: Márquez-Artavia et al. (2017). 
de frecuencias complejas, de la forma: $\omega=\omega_{r}-i \omega_{i}$, donde $\omega_{r}$ es la parte real de la frecuencia asociada a la oscilación de la onda mientras que $\omega_{i}$ es la parte imaginaria que es la que introduce la inestabilidad (Chandrasekhar, 2013), debido a la forma matemática que tiene la solución de onda,

$$
u=\tilde{u} e^{i \omega t} \Rightarrow \tilde{u} e^{i \omega_{r} t} e^{\omega_{i} t},(4)
$$

en este caso $u$ y $\tilde{u}$ corresponden a la velocidad y su amplitud, por ejemplo. Hay que aclarar que la inestabilidad solo se presenta si el campo magnético tiene una cierta intensidad, si la intensidad es débil la inestabilidad no se presentará (Malkus, 1967). Este fenómeno corresponde únicamente a las ondas magnéticas de Rossby, si el campo magnético está ausente no encontraremos frecuencias complejas, además de que los otros tipos comunes de ondas en fluidos como las ondas magnéticas de Kelvin o las ondas magneto-inerciales de gravedad no presentan inestabilidad. En la naturaleza hay muchas manifestaciones de inestabilidad, por ejemplo en las nebulosas que dan origen a las estrellas, el inicio de la acumulación de masa por atracción gravitatoria puede deberse a una inestabilidad.

En este apartado enunciamos las características de las ondas magnéticas de Rossby, en la siguiente sección se propone que los ciclos solares son manifestaciónes de estas ondas.

\section{Los ciclos solares y las ondas magnéticas de Rossby}

Desde la antigüedad el Sol ha sido objeto de múltiples y cuidadosas observaciones, existen registros de observaciones desde la época de Galileo Galilei (1564-1642). En especial, las observaciones de manchas solares de Samuel Heinrich Schwabe (1789-1875), quien descubrió que el número total de manchas solares varía periódicamente, al cambiar de un máximo a un mínimo y de nuevo a un máximo con un periodo de 11 años (Lang, 1995). Este es el ciclo de Schwabe.

Además, el ciclo magnético solar es de 22 años (Lang, 1995). Las manchas solares aparecen a cierta latitud alrededor de $\sim 40^{\circ}$, y se van generando cada vez más cerca del Ecuador, al completarse los once años la polaridad del campo magnético del Sol se invierte, y comienza el ciclo de Schwabe de nuevo, pero con la polaridad opuesta. Este ciclo de 22 años, se denomina ciclo de Haly (ver Figura 5).

Durante los máximos de actividad, se producen más eyecciones de masa coronal (CME, por sus siglas en inglés) donde una gran cantidad de materia y energía es expelida del Sol. También se incrementa el número de fulguraciones y todo tipo de explosiones violentas, que generan las perturbaciones del "viento solar" que permea todo el sistema solar y aún más allá (Kahler, 1992).

Las partículas más energéticas del viento solar son esparcidas por todo el sistema solar, que alcanzan la Tierra en un lapso de tiempo que va desde unas horas hasta unos pocos días lo que produce fenómenos como las auroras boreales, al interactuar las partículas con las moléculas que se encuentran en las capas superiores de la atmósfera. Así también el viento solar tiene un efecto negativo sobre los satélites artificiales que rodean la Tierra; la radiación y las partículas pueden dañarlos incluso permanentemente. En algunos casos, las tormentas solares más intensas han producido daños en centrales eléctricas en países con altas latitudes geográficas, como Canadá (Board y National Research Council, 2012). 


\section{DAILY SUNSPOT AREA AVERAGED OVER INDIVIDUAL SOLAR ROTATIONS}
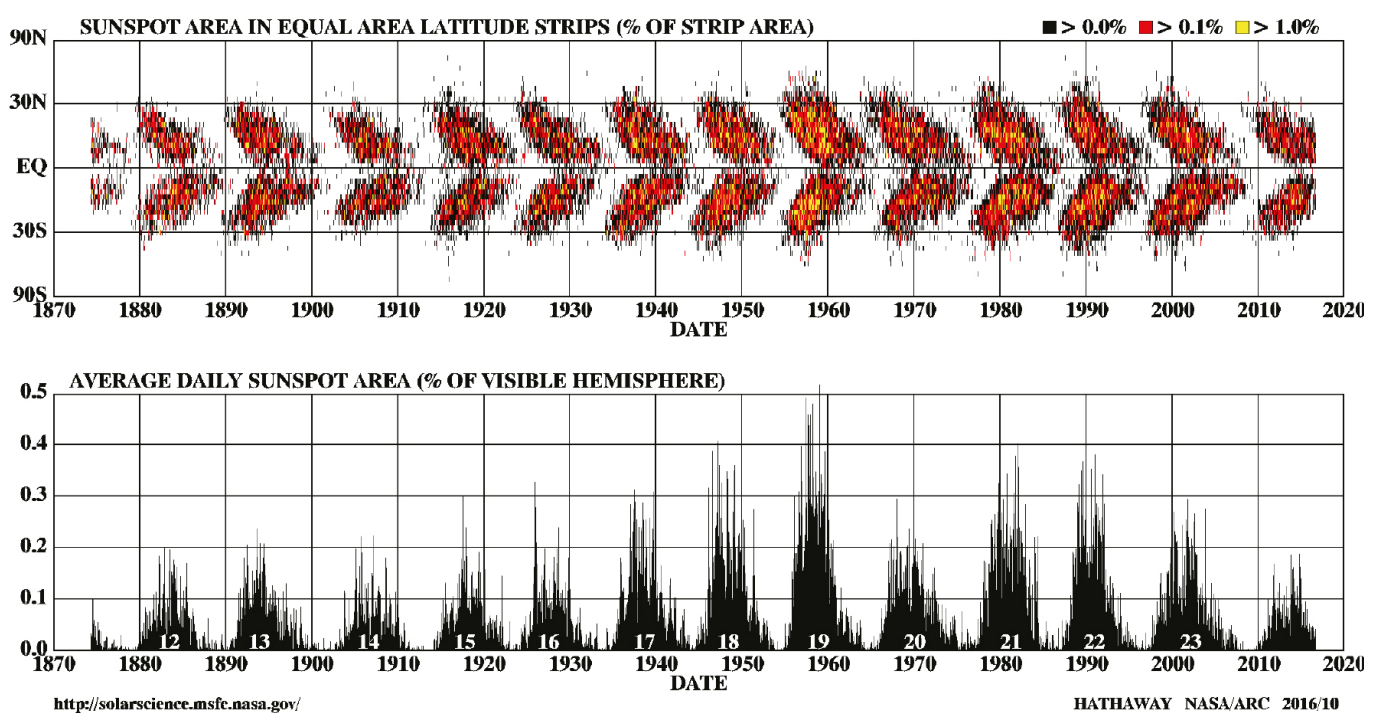

Figura 5. Diagrama de mariposa que muestra los ciclos de manchas solares desde 1870 hasta la actualidad.

Fuente: Extraído de: National Aeronautics and Space Administration (2018)

Los máximos y mínimos en la cantidad de manchas solares, mostrados en la Figura 5, tienen también otras periodicidades donde son mucho más intensos o mucho menos intensos. Por ejemplo el ciclo de Gleissberg de 87-95 años de duración o el ciclo de Suess con un periodo de 210 años.

Al analizar los isótopos radiogénicos de C-14 y Be-10 que están presentes en los troncos de los árboles y en el hielo y son sensibles a la radiación solar, se han encontrado otras periodicidades, como el ciclo de 2300 años.

Las periodicidades de tipo Rieger también son de largo periodo, Zaqarashvili et al. (2010a, 2015) han encontrado a partir de medidas de concentraciones de isótopos radiogénicos una serie de máximos de actividad con una secuencia de 1000, 500, 350, 200 y 100 años que han sido asociados por estos autores a un conjunto de modos magnéticos lentos de Rossby.

Al igual que esta manifestación, otras investigaciones (Lou et al., 2003) han detectado periodicidades para las CME (actividades coronales), llamaradas ("flares", actividades cromosféricas), y otros fenómenos de la actividad solar, que están relacionados indirectamente con el índice-Ap, el cual es uno de los indicadores de la actividad geomagnética. En la Tabla 1 se ha resumido la información anterior.

Tabla 1

Lista de cuasiperiodos encontrados (en datos desde el 5 de febrero de 1999 al 10 de febrero del 2003)

\begin{tabular}{|l|l|l|l|l|l|l|l|l|l|l|l|l|}
\hline \multicolumn{1}{|c|}{ Tipo de fenómeno } & \multicolumn{10}{c|}{ Cuasiperiodos en días } \\
\hline CME & 1101 & 358 & 272 & 196 & 111 & 100 & 66 & 61 & 57 & 36 & 33 & 21 \\
\hline Llamaradas ("flares") & 1505 & 259 & 157 & 122 & 98 & 68 & 64 & 42 & 39 & 34 & & \\
\hline Ap & 941 & 364 & 273 & 187 & 138 & 91 & 61 & 28 & 27 & 25 & 24 & 23 \\
\hline
\end{tabular}

Nota: Fuente Lou et al.( 2003). 
La actividad geomagnética se registra continuamente a través de magnetrómetros ubicados en todo el mundo, estos miden el flujo de protones que provienen del Sol. El índice-Ap muestra el promedio diario de la actividad geomagnética. Un índice-Ap grande $(>30)$ puede considerarse una tormenta geomagnética y si se prolonga puede causar problemas en satélites y otras tecnologías, lo que ocasiona graves daños materiales.

Una tormenta geomagnética es una fuerte perturbación del campo magnético terrestre producida por intensas eyecciones de partículas ionizadas que se forman en la corona solar, alcanzan la tierra 24-36 horas después de que ocurre la explosión en el Sol, pueden durar horas o días, y su mayor intensidad se experimenta en las latitudes boreales o australes, (Lang, 1995).

Estas son manifestaciones del clima espacial, que se define como las condiciones de cualquier medio (Sol, medio interplanetario, campo magnético, atmósfera y superficie terrestre) que son influidas por la interacción entre el Sol y la Tierra (Schwenn, 2006).

Varios autores (Márquez et al., 2017, McIntosh et al., 2017, Zaqarahvili et al., 2010b, 2018) han considerado muchas de estas periodicidades asociadas a las ondas magnéticas de Rossby, unas relacionadas con las ondas lentas y otras con las rápidas. En la siguiente sección se hará un análisis cualitativo de las periodicidades y su comparación con las observaciones.

\section{Metodología}

Este trabajo se fundamenta en una revisión bibliográfica, principalmente de libros y artículos científicos, se siguen de cerca las investigaciones de Zaqarashvili y otros investigadores (2007-2018), quienes han desarrollado el modelo matemático en cuestión, así como los textos más tradicionales de Gilman (2000) y Dikpati, et al. (2001).

Uno de los criterios de selección de los artículos ha sido la fecha de publicación, que trata de incluir nuevos resultados e ideas actualizadas.

El análisis de resultados está basado en el desarrollo teórico y numérico del modelo 2D de la capa delgada de fluido para un campo magnético toroidal propuesto por Márquez-Artavia et al. (2017), que es tiene la solución más completa y sistemática para el problema propuesto por Zaqarashvili, et al. (2009).

Para la discusión de los resultados, se utilizan las fórmulas del trabajo previo (Márquez-Artavia, et al., 2017) para calcular las frecuencias y los periodos de las ondas magnetohidrodinámicas, luego se comparan con las observaciones registradas por otros investigadores y aún registros de la actividad solar encontrados comúnmente en la literatura.

Los registros de observaciones se buscaron en muchas fuentes, en especial artículos científicos, en este caso Lou, et al. (2003).

\section{Análisis de resultados}

Las periodicidades de muy largo periodo y de frecuencias pequeñas, como el ciclo de Gleissberg, el ciclo de Suess, las periodicidades tipo Rieger, incluso el ciclo de 2300 años, con duraciones superiores a los cien años, podrían estar relacionadas con ondas magnéticas lentas de Rossby, que al producirse en el interior del Sol, en la tacoclina, son capaces de levantar masas de fluido a través de la zona convectiva y manifestarse en la superficie, como cambios en el flujo magnético, que posibilitan la formación de regiones activas, como lo han sugerido Dikpati, et al. (2017). 
La ecuación (2) que describe la frecuencia para estas ondas lentas es directamente proporcional al valor de $B_{0}^{2}$, el valor del campo magnético en la tacoclina, lo que hace que los valores de las frecuencias y periodos sean muy sensibles al valor numérico del campo. Por lo que un campo magnético solar en esta delgada capa puede tener un valor del orden de $10^{-3} T$, (Márquez-Artavia, 2017), para corresponder a periodicidades de muy largo plazo. Este es un valor de $B_{0}$ mucho menor del que se ha sugerido en la literatura.

Los otros eventos de la actividad solar con una duración de días, se pueden asociar a las ondas magnéticas rápidas de Rossby, por ejemplo: la actividad solar de la Tabla 1. En este caso las llamaradas, CME, y el índice-Ap muestran un conjunto de periodos que van desde cientos de días a unas decenas. En el caso de la fórmula (1), se predice un conjunto de ondas con periodos de 27, 80, 161, 269, 404 días,... para $(m=1, n=1,2,3,4,5, \ldots, \ldots$ respectivamente). Aunque los valores no poseen el mismo espaciamiento temporal de los valores de la Tabla 1, se encuentran en el mismo orden de magnitud.

Una particularidad que se deriva de este resultado es que la formula (1) demuestra que la frecuencia es directamente proporcional a $\Omega_{0}$, la velocidad de rotación del Sol, solo depende de este parámetro. Además, las estrellas presentan rotación diferencial, su tasa de rotación es diferente con la latitud, esta variación en el valor numérico de la rotación puede introducir diferencias en los periodos que van desde $1,2,4,6$, 8 días, ...(para $n=1,2,3,4,5, \ldots .$.$) . Este$ hecho es un indicador de que el introducir la rotación diferencial en un nuevo modelo matemático va a contribuir a mejores y más reales resultados para las ondas magnéticas rápidas de Rossby.

Otro dato importante y clave es el ciclo de Haly, este tiene la particularidad de que las manchas solares aparecen en una banda de latitud de $\pm 40^{\circ}$ y conforme avanza el ciclo se confinan al Ecuador, en esta característica se asemeja a una onda magnética rápida de Rossby.

Las ondas anómalas de Rossby son de periodos muy largos, que podrían abarcar millones de años. No existe aún una observación o cálculo de tan largo alcance para esta estrella, por lo que no es posible asociarle algún sentido físico por el momento. Este es un modo que debe su existencia al campo magnético, se ha notado que la ecuación (3) depende del campo magnético a la cuarta potencia, $B_{0}^{4}$, que generalmente tiene valores menores que $1 \mathrm{~T}$, es de esperarse que las ondas anómalas sean extremadamente lentas, las observaciones con periodos tan largos todavía no se han registrado.

Otros modelos más complejos de generación de ondas en el interior del Sol han sido desarrollados (Schecter et al., 2001, Dikpati et al., 2001), sin embargo, no existe certeza del tipo de actividad solar sea causada por estas ondas y qué implicaciones puede tener en el clima espacial. Este es solo el comienzo.

\section{Conclusiones}

El propósito de este artículo es valorar si existe una relación entre las observaciones de los ciclos solares y las ondas magnéticas de Rossby, ambos como fenómenos de origen magnético. Se ha identificado que los ciclos de muy largo periodo se asemejan a las ondas magnéticas lentas de Rossby mientras que los ciclos de periodos de días a meses son más similares a las ondas magnéticas rápidas de Rossby. 
La principal implicación teórica de estas ideas es que las ondas magnetohidrodinámicas pueden ser la clave para descifrar la dinámica del Sol y muchos aspectos que aún no se comprenden. Esta discusión de los resultados encontrados por Márquez-Artavia, et al. (2017) pretenden explicar a profundidad las propiedades de las ondas de Rossby, de forma concisa, y en su totalidad.

Actualmente el diagnóstico del clima espacial ha avanzado de manera significativa, en especial, la predicción de los términos de corto o largo plazo con buena exactitud. Sin embargo, una compresión más profunda del origen y las propiedades de la actividad solar a muy largo plazo puede mejorar los pronósticos (Toledo, 2013).

Nuevas investigaciones deben de ser desarrolladas en el área, un paso importante sería realizar un análisis que involucre la rotación diferencial, que es una característica de las estrellas. Introducir un campo magnético dipolar es una meta realizable que puede ilustrar otras propiedades de las ondas magnetohidrodinámicas, también plantear un nuevo modelo 3D para incluir la coordenada vertical, conducirá a otras soluciones que pueden representar de una forma más real el sistema en cuestión.

Comprender a cabalidad la dinámica de las ondas magnetohidrodinámicas es un paso adelante en el camino al pronóstico del clima espacial, que traerá un gran beneficio a la comunidad global.

\section{Agradecimientos o reconocimientos}

Este trabajo fue elaborado en el marco del proyecto de investigación del Laboratorio de Oceanografía y Manejo Costero SIA 0445-14 y como parte del proyecto de tesis de la beca: JB-C0596-2013 de la Universidad Nacional de Costa Rica.

\section{Referencias}

Board, S. S., \& National Research Council. (2012). The Effects of Solar Variability on Earth's Climate: A Workshop Report. National Academies Press.

Chandrasekhar, S. (2013). Hydrodynamic and hydromagnetic stability. Courier Corporation.

Dikpati, M., Cally, P. S., McIntosh, S. W., \& Heifetz, E. (2017). The Origin of the "Seasons" in Space Weather. Scientific reports, 7(1), 14750. doi: https://doi.org/10.1038/ s41598-017-14957-x

Dikpati, M., \& Gilman, P. A. (2001). Analysis of hydrodynamic stability of solar tachocline latitudinal differential rotation using a shallow-water model. The Astrophysical Journal, 551(1), 536. http://dx.doi.org/10.1086/320080.

Gilman, P. A. (2000). Magnetohydrodynamic "shallow water" equations for the solar tachocline. The Astrophysical Journal Letters, 544(1), L79. https://doi.org/10.1086/317291

Gilman, P. A., \& Dikpati, M. (2002). Analysis of instability of latitudinal differential rotation and toroidal field in the solar tachocline using a magnetohydrodynamic shallow-water model. I. Instability for broad toroidal field profiles. The Astrophysical Journal, 576(2), 1031. http://dx.doi.org/10.1086/341799

Heng, K., \& Spitkovsky, A. (2009). Magnetohydrodynamic shallow water waves: linear analysis. The Astrophysical Journal, 703(2), 1819. http://dx.doi. org/10.1088/0004-637X/703/2/1819

Hughes, D. W., Rosner, R., \& Weiss, N. O. (Eds.). (2007). The Solar Tachocline. Cambridge University Press. doi: https://doi.org/10.1017/ CBO9780511536243

Kahler, S. W. (1992). Solar flares and coronal mass ejections. Annual Review of Astronomy and Astrophysics, 30(1), 113-141. http://dx.doi. org/10.1146/annurev.aa.30.090192.000553

Kessler, W. S. (2006). The circulation of the eastern tropical Pacific: A review. Progress in Oceanography, 69(2-4), 181-217. http://dx.doi. org/10.1016/j.pocean.2006.03.009

Lang, K. R. (1995). Sun, earth and sky. Earth, Moon, and Planets, 70(1-3), 1-20. doi: https://doi. org/10.1007/978-3-642-57852-6

Lou, Y. Q., Wang, Y. M., Fan, Z., Wang, S., \& Wang, J. X. (2003). Periodicities in solar coronal mass 
ejections. Monthly Notices of the Royal Astronomical Society, 345(3), 809-818. https://doi. org/10.1046/j.1365-8711.2003.06993.x

Malkus, W. V. (1967). Hydromagnetic planetary waves. Journal of Fluid Mechanics, 28(4), 793-802. https://doi.org/10.1017/ s0022112067002447

Márquez-Artavia, X. (2017). Global Magnetohydrodynamic Waves in Stably Stratified Rotating Layers. (Tesis doctoral). Universidad de Leeds, Inglaterra.

Márquez-Artavia, X., Jones, C. A., \& Tobias, S. M. (2017). Rotating magnetic shallow water waves and instabilities in a sphere. Geophysical \& Astrophysical Fluid Dynamics, 111(4), 282-322. https://doi.org/10.1080/03091929.2 017.1301937

McIntosh, S. W., Cramer, W. J., Marcano, M. P., \& Leamon, R. J. (2017). The detection of Rossby-like waves on the Sun. Nature Astronomy, 1(4), 0086. https://doi.org/10.1038/ s41550-017-0086.

Miesch, M. S. (2005). Large-scale dynamics of the convection zone and tachocline. Living Reviews in Solar Physics, 2(1), 1. https://doi. org/10.12942/lrsp-2005-1

National Aeronautics and Space Administration. (2018). Solar Physics. [página web]. Recuperado de: http://solarscience.msfc.nasa.gov/.

Schecter, D. A., Boyd, J. F., \& Gilman, P. A. (2001). "Shallow-water" magnetohydrodynamic waves in the Solartachocline. The Astrophysical Journal Letters, 551(2), L185. http://dx.doi. org/10.1086/320027

Schwenn, R. (2006). Space weather: The solar perspective. Living Reviews in Solar Physics, 3(1), 2. https://doi.org/10.12942/lrsp-2006-2

Solanki, S. K., Inhester, B., \& Schüssler, M. (2006). The solar magnetic field. Reports on Progress in Physics, 69(3), 563. http://dx.doi. org/10.1088/0034-4885/69/3/R02
Tobias, S. M. (2005). The solar tachocline: Formation, stability and its role in the solar dynamo. Fluid dynamics and dynamos in astrophysics and geophysics, 1, 193. https://doi. org/10.1017/cbo9780511536243.014

Toledo, J. C. (2013) Variabilidad solar y clima terrestre. Nasa: Ciencia beta. Recuperado de: https://ciencia.nasa.gov/ ciencias-especiales/08jan_sunclimate

Zaqarashvili, T. (2018). Equatorial magnetohydrodynamic shallow water waves in the solar tachocline. The Astrophysical Journal, 856(1), 32. https://doi.org/10.3847/1538-4357/aab26f

Zaqarashvili, T. V., Carbonell, M., Oliver, R., \& Ballester, J. L. (2010a). Magnetic Rossby waves in the solar tachocline and Rieger-type periodicities. The Astrophysical Journal, 709(2), 749. http://dx.doi. org/10.1088/0004-637X/709/2/749

Zaqarashvili, T. V., Carbonell, M., Oliver, R., \& Ballester, J. L. (2010b). Quasi-biennial oscillations in the solar tachocline caused by magnetic Rossby wave instabilities. The Astrophysical Journal Letters, 724(1), L95. http://dx.doi. org/10.1088/2041-8205/724/1/L95

Zaqarashvili, T. V., Oliver, R., \& Ballester, J. L. (2009). Global shallow water magnetohydrodynamic waves in the solar tachocline. The Astrophysical Journal Letters, 691(1), L41.http:// dx.doi.org/10.1088/0004-637X/691/1/L41

Zaqarashvili, T. V., Oliver, R., Hanslmeier, A., Carbonell, M., Ballester, J. L., Gachechiladze, T., \& Usoskin, I. G. (2015). Long-term variation in the Sun's activity caused by magnetic Rossby waves in the tachocline. The Astrophysical Journal Letters, 805(2), L14. http://dx.doi. org/10.1088/2041-8205/805/2/L14

Zeilik, M., \& van Panhuys Smith, E. (1987). Introductory astronomy and astrophysics. Philadelphia: Saunders College Pub., c1987. 2nd ed.

\section{(C) $(1 \Theta \Theta$}

Descifrando el clima espacial: ciclos solares y ondas magnéticas de Rossby (Xiomara Márquez-Artavia) por Revista Uniciencia se encuentra bajo una Licencia CreativeCommons Atribución-NoComercial-SinDerivadas 3.0 Unported. 